Aufgrund von Umstellungen im Herstellungsprozess des Verlags gibt Frau Annelie Meisenheimer zum Ende dieses Monats die langjährige Heftbetreuung der Zeitschrift für Betriebswirtschaft in andere Hände. Für die erfolgreiche und engagierte Zusammenarbeit danken ihr die Herausgeber der Zeitschrift ganz herzlich. Für ihre neuen Aufgabengebiete im Verlag wünschen wir ihr viel Erfolg und alles Gute für die Zukunft.

Die vier Artikel dieses Heftes leisten Beiträge zu den Gebieten der Corporate Governance, der Steuerlehre, der Lagerhaltung und des Kostenmanagements. Der letzte Beitrag ist ein Übersichtsartikel.

\title{
Personelle Unternehmensverflechtung und Vorstandsgehälter
}

Balsmeier und Peters untersuchen in ihrem empirischen Beitrag, welche Auswirkung die Wahrnehmung von Aufsichtsratsmandaten in anderen Unternehmungen auf die Vorstandvergütung in den entsendenden Unternehmungen hat. Zugleich soll überprüft werden, inwieweit die Erlangung zusätzlicher Aufsichtsratsmandate durch die Leistung der Vorstände in den entsendenden Unternehmungen erklärt werden kann. Nach theoretischen Erörterungen, ob die Prinzipal-Agenten-Theorie für diese Zusammenhänge als Erklärungsansatz in Frage kommen kann, und nach der vergleichsweisen Erörterung vorliegender Studien aus dem US-amerikanischen Bereich formulieren die Autoren einen entsprechenden Regressionsansatz. Die der Regression zugrunde liegenden Daten wurden aus den Geschäftsberichten der 100 wertschöpfungsstärksten deutschen Unternehmen gewonnen. In den linearen Regressionsansatz zur Erklärung der Vorstandvergütungen pro Kopf in Abhängigkeit der personellen Verflechtungen von Vorständen mit Aufsichtsräten anderer Unternehmungen gehen als erklärende Variablen die Anzahl der Verflechtungen, die Unternehmensgröße, die Eigenkapitalrendite, die Anzahl der Mitglieder im Aufsichtsrat sowie das Ausmaß des Streubesitzes an den Aktien ein. Dabei zeigt sich, dass die Anzahl der personellen Verflechtungen, die Unternehmensgröße und das Ausmaß des Streubesitzes einen positiven Einfluss auf die Höhe der Vorstandsvergütung pro Kopf haben. Der Einfluss der Eigenkapitalrendite fällt gering aus; im Hinblick auf die Anzahl der Mitglieder im Aufsichtsrat kann kein signifikanter Zusammenhang festgestellt werden. Die weitergehende Frage, inwieweit die hergeleiteten Ergebnisse durch bessere Verhandlungspositionen oder stärkere Leistung der entsendeten Vorstände erklärbar sind, führt nicht zu einem einheitlichen Ergebnis. Insofern kann auch die Hypothese nicht bestätigt werden, dass Vorstände leistungsstärkerer Unternehmen eher in Aufsichtsräte anderer Unternehmungen berufen werden. So vertreten die Autoren auch die 
Meinung, dass für den Fall einer fehlenden Evidenz überdurchschnittlicher Leistungen verflochtener Vorstände Mehrfachmandate kritisch beurteilt werden müssen.

\section{Auswirkungen der Zinsschranke auf die Bewertung des Tax Shield nach der Unternehmensteuerreform}

In seinem methodisch ausgerichteten Beitrag beschäftigt sich Stöckl mit der Frage, welche Auswirkungen die Zinsschranke nach der Reform der Unternehmensbesteuerung auf die Bewertung der Tax Shields der Fremdkapitalfinanzierung und damit schließlich auch auf die Unternehmensbewertung nach der Unternehmensteuerreform von 2008 besitzt. Als Ansatz für eine adäquate und verlässliche Unternehmensbewertung schlägt der Autor einen modifizierten Ansatz zum Adjusted Present Value-Ansatz vor. Der Ansatz erlaubt es, Tax Shields bestimmter Jahre in die Teile zu zerlegen, die aus den periodenmäßigen Fremdkapitalkosten entstanden sind, und solche, welche aufgrund von Zinsvorträgen generiert werden. Der Verfasser versucht, mit dem vorgestellten Modell die Vorzüge des Ansatzes der Weighted Average Costs of Capital und des Standard-Ansatzes des Adjusted Present Value miteinander zu verbinden und damit zugleich die Discounted Cashflow-Methode zur Unternehmensbewertung zu ergänzen. Die vom Verfasser entwickelte neue Reaktionsformel ermöglicht es, durch angepasste Eigenkapitalkosten im Rahmen eines modifizierten Adjusted Present Value-Ansatzes eine risikoadäquate Bewertung von Unternehmungen vorzunehmen. Zu diesem Zweck werden vier Fälle unterschieden und durch entsprechende numerische Beispiele in ihren Auswirkungen untersucht. Dabei geht es einmal darum, dass die Zinsschranke für das Unternehmen aufgrund seiner Ergebnissituation direkt wirkt und dabei entweder kein Zinsvortrag aus Vorperioden vorliegt oder aber doch in Ansatz gebracht werden kann. In den beiden anderen Fällen, in denen jeweils ein Zinsvortrag vorliegt, unterscheidet der Verfasser danach, ob die Zinsschranke indirekt oder aber überhaupt nicht wirkt. Die Risikoeigenschaften des entsprechenden Tax Shields werden auf der Grundlage einer zweiperiodigen Binomialverteilung eingeschätzt. Nach entsprechenden Äußerungen in der einschlägigen Literatur muss man wohl davon ausgehen, dass in Deutschland 500 bis 1.500 Kapitalgesellschaften von einer derartigen Zinsschrankenauswirkung betroffen sind. Die methodischen Überlegungen des Verfassers erhalten somit eine erheblich empirische Relevanz.

\section{Flexibilität von Strategien zur Ersatzteilversorgung}

Die modellgestützte Flexibilitätsanalyse von Strategien zur Ersatzteilversorgung in der Nachserienphase ist Gegenstand der Ausführungen von Kleber und Inderfurth. Auf der Grundlage der Formulierung eines mehrperiodigen Lagerhaltungsmodells werden unterschiedliche Beschaffungsoptionen und deren Kombinationen im Hinblick auf ein optimales Bestandsmanagement von Ersatzteilen langlebiger Industrie- und Konsumgüter untersucht. Dabei sind bezüglich der Flexibilität der Ersatzteilversorgung die Mengenflexibilität, die Zeitflexibilität und die Bestandsflexibilität von besonderer Bedeutung. Als Versorgungsoptionen betrachten die Autoren die Abschlusslosbildung zu Serienbedin- 
gungen, die Abschlusslosbildung und Nachproduktion in kleineren Losen, die Abschlusslosbildung und die Aufarbeitung von Altprodukten sowie die Kombination aus allen drei Möglichkeiten. Die flexibilitätsbedingten Erfolgsbeiträge dieser Strategien werden dann durch ihren relativen Kostenvorteil gegenüber der ausschließlichen Nutzung eines Abschlussloses bestimmt. In dem von den Autoren formulierten Lagerhaltungsmodell werden die erwarteten Gesamtkosten minimiert, wobei die Höhe des Abschlussloses sowie die Nachproduktionsmengen und Aufarbeitungsmengen der verschiedenen Perioden die Entscheidungsvariablen sind. Für verschiedene Nachfrageszenarien des Ersatzteilbedarfs und unterschiedliche Rückflussströme an Altprodukten lassen sich dann auf der Grundlage der gesetzten Kostenparameter die Flexibilitätsvorteile der unterschiedlichen Beschaffungsoptionen bestimmen. Dabei zeigt sich aufgrund der modellgestützten Berechnungen, dass die Nachproduktion den höchsten Erfolgsbeitrag ausweist, sofern die Beachtung von Mindestproduktionsmengen diese Flexibilität nicht einschränkt. Die Altteileaufarbeitung erscheint dem gegenüber weniger erfolgversprechend, da sie wegen der Unbestimmtheit der zurückfließenden Altteile nur eine eingeschränkte Mengenflexibilität besitzt. Bei niedrigen Stückkosten kann die Aufarbeitung allerdings dennoch attraktiv sein. Die Vorteilhaftigkeit der Nachproduktion und der Aufarbeitung gegenüber der Abschlusslosbildung nimmt mit zunehmenden Stückkosten ab. Allerdings - so zeigen die Autoren - bleiben beide Beschaffungsoptionen sinnvolle Ergänzungen zur Ersatzteilversorgung in Form der alleinigen Bildung eines Schlussloses. Weitere Kombinationen der Versorgungsstrategien versprechen dagegen nur noch einen geringfügigen zusätzlichen Erfolgsbeitrag. Die Streuung der Altproduktrückflüsse hat einen geringeren Einfluss auf die Gesamtkosten als die spezifischen Beschaffungskosten der Optionen und die Höhe der Lagerkosten. Für die Wahl der richtigen Versorgungsoption ist das Ausmaß der Nachfragestreuung bedeutungsvoller als die Streuung der Altproduktrückflüsse.

\section{Kostenmanagement}

In seinem Übersichtsaufsatz nimmt Himme eine Bestandsaufnahme und kritische Beurteilung der empirischen Forschung zum Gebiet des Kostenmanagement vor. Wenn die empirischen Beiträge im Vergleich zu den konzeptionellen Arbeiten gemessen an der Anzahl deutschsprachiger Veröffentlichungen zum Thema Kostenmanagement auch noch relativ gering sind, so liegen doch seit Beginn der 90er Jahre des vorigen Jahrhunderts bis zum Jahre 2007 rund 40 empirische Arbeiten vor, über deren Ergebnisse der Autor einen systematischen Überblick geben möchte. Die Aussagen werden anhand eines Bezugsrahmens zum Kostenmanagement geordnet, der im Hinblick auf die inhaltliche Gliederung von Kostenmanagementsystemen zwischen Aufgaben, Instrumenten, Institutionen und Objekten unterscheidet und zudem die Auswirkungen unternehmensinterner und unternehmensexterner Faktoren auf die Gestaltung von Kostenmanagementsystemen erfasst. Insbesondere sollen durch den systematischen Überblick Antworten auf die Fragen gegeben werden, welche empirischen Befunde zum Kostenmanagement aktuell vorliegen, welche die inhaltlichen und methodischen Schwerpunkt der empirischen Arbeiten sind, wie die Erfolgsbeiträge der Arbeiten zu beurteilen sind und welcher weitere Forschungsbedarf besteht. Wenn die empirischen Arbeiten auch ein hohes Maß an Heterogenität auf- 
weisen, so lässt sich doch feststellen, dass die Arbeiten zum Target Costing vorherrschend sind, auch wenn dessen Implementierungsaufwand im Vergleich zu anderen Instrumenten des Kostenmanagements relativ hoch ist. Der Erfolg der Anwendung von Kostenmanagementmaßnahmen wird nur mäßig eingeschätzt. Viele empirische Untersuchungen lassen einen konzeptionellen geschlossenen Bezugsrahmen vermissen. Verhaltensorientierte Aspekte des Kostenmanagements werden meist vernachlässigt. So bedarf es für die zukünftigen Forschungen insbesondere einer Bestimmung von Einflussfaktoren für ein erfolgreiches internes und unternehmensübergreifendes Kostenmanagement.

\section{Das Oktober-Heft}

Das nächste Heft enthält vier Beiträge, von denen wiederum einer einen Übersichtartikel darstellt. Die vier Arbeiten kommen aus den Bereichen der Personalwirtschaft, der Finanzierung und der Betriebswirtschaftlichen Steuerlehre.

Entorf, Gattung, Möbert und Pahlke untersuchen den Einfluss von Aufsichtsratsvernetzungen auf die Entwicklung der Vorstandsbezüge von DAX-Unternehmen. Der Beitrag ergänzt die Überlegungen von Balsmeier und Peters im vorliegenden Heft. Die Autoren kommen ebenfalls zu dem Ergebnis, dass die Vorstandsgehälter eines Unternehmens mit der Zahl der wahrgenommenen Aufsichtsratsmandate in anderen Unternehmen steigen. Allerdings zeigen die Ergebnisse von Entorf u.a., dass die Vorstandsgehälter mit der Zahl fremder Vorstände im eigenen Aufsichtsrat sinken. Der Wechsel eines ehemaligen Vorstandsvorsitzenden an die Spitze des Aufsichtsrats führt nicht zu signifikant höheren Vorstandsgehältern.

Die Auswirkungen der Berücksichtigung liquider Assets in der Portfoliooptimierung sind Gegenstand der Analyse von Diepold und Dzienziol. Anhand eines einfachen Modells werden die grundlegenden Unterschiede zu einer Optimierung mit lediglich liquiden Assets nachgewiesen. So kann für den Fall zufälliger Liquiditätsanforderungen eine Aufteilung des Portfolios auf liquide und illiquide Assets optimal sein.

Die Verlustnutzung von Kapitalgesellschaften bei Umwandlungen ist Gegenstand einer ökonomischen Vorteilhaftigkeitsanalyse von Brähler, Goettsche und Rauch. Die Autoren analysieren die steuerlichen Auswirkungen der im Rahmen von Umwandlungen lediglich indirekten Verlustnutzungsmöglichkeit durch Aufstockung der Wirtschaftsgüter sowie der Mindestbesteuerung.

Die Darstellung der Forschungsziele der Betriebswirtschaftlichen Steuerlehre und ihre kritische Würdigung sind Inhalt des Übersichtsbeitrags von Schmiel. Die Autorin erhebt dabei den Einwand, dass neoklassisch-modelltheoretische Steuerwirkungshypothesen wegen ihrer realitätsfernen Prämissen nicht geeignet sind, das Handeln von Steuerpflichtigen zu erklären. Sie plädiert deswegen dafür, in der Steuerwirkungslehre die tatsächlichen Einflüsse von Steuern auf das Handeln von Steuerpflichtigen losgelöst von präskriptiven Entscheidungsmodellen und deren Rationalitätsvorstellungen zu untersuchen.

Günter Fandel

Editor-in-Chief

E-Mail: ZFB@FernUni-Hagen.de 